\title{
Polarimetry - from basics to precision
}

\author{
I. Keshelashvili; ${ }^{* 1}$, F. Müller ${ }^{1,2}$, D. Mchedlishvili ${ }^{3,4}$, D. Shergelashvili ${ }^{4}$ \\ on behalf of the JEDI collaboration \\ ${ }^{1}$ Institute of Nuclear Physics, Forschungszentrum Jülich, 52425 Jülich, Germany \\ ${ }^{2}$ Physics Institute III B, RWTH Aachen, Otto-Blumenthal-Strasse, 52074 Aachen, Germany \\ ${ }^{3}$ High-Energy Physics Institute, Tbilisi State University, 0186 Tbilisi, Georgia \\ ${ }^{4}$ SMARTIEDM_Lab, Tbilisi State University, 0179 Tbilisi, Georgia \\ E-mail: i.keshelashviliefz-juelich.de
}

In all experiments involving polarized beams or/and targets, the determination of the degree of polarization by asymmetry measurements is of prime importance. Over the years, so-called analyzing powers were extracted, e.g., for various reactions for well-defined beam/target polarizations as a function of the reaction energy. Reciprocally, the polarization can be deduced precisely from measured asymmetries if the analyzing power is known. In practice, the task may become complicated by the required unambiguous identification of the reaction channel. In cases where the statistics are limited, such as, e.g., in the planned EDM (Electric Dipole Moment)-search for charged particles in storage rings, polarimetry becomes a real challenge. In this overview, different aspects of possible polarimetry concepts, the efficient reaction selection, and the quasi non-invasive scattering targets will be discussed. Also, the recent progress achieved by the JEDI collaboration using polarized deuteron beams of COSY-Jülich will be presented.

XVII International Workshop on Polarized Sources, Targets \& Polarimetry

16-20 October 2017

Kaist, South Korea

${ }^{*}$ Speaker. 


\section{Introduction}

The JEDI (Jülich Electric Dipole moment Investigations) collaboration [1] is formed with the aim to search the EDM (Electric Dipole Moment) of the elementary charged hadrons like protons and deuterons. The COoler SYnchrotron (COSY) [2] is ideally suited for the first steps toward storage ring EDM search. It can be successfully used for the protons beam polarization and is currently intensively utilized for the deuteron beam polarization study. During last several years, a significant breakthrough has been made using COSY. A new method has been developed for a continuous determination of the spin tune in COSY and implications for precision experiments were investigated [3]. It was demonstrated to have extremely stable spine tune in the orders of $10^{-10}$ during 100 second accelerator cycles. In the consequent study, the record results of in-plane polarization lifetime with $970 \mathrm{MeV} / \mathrm{c}$ deuterons in the COSY ring has been reached [4]. Equipping the COSY with the feedback loop between the polarimeter and the spin precession driver devices (like RF solenoid or RF cavity) brought us to a new level where the beam polarization vector phase can be locked [5] for the certain value. Stabilizing the spin tune in the storage ring and measuring it with the unprecedented stability brought us to map a spin tune to probe the spin dynamics in storage rings [6]. Starting from 2017 we installed the dedicated waveguide, RF Wien Filter [7], specifically designed for the EDM search in the COSY ring. RF Wien Filter measurement will lead us to first precursor EDM measurement [8] where the method will be the "partially frozen spin" effect [9].

\section{Polarimeter}

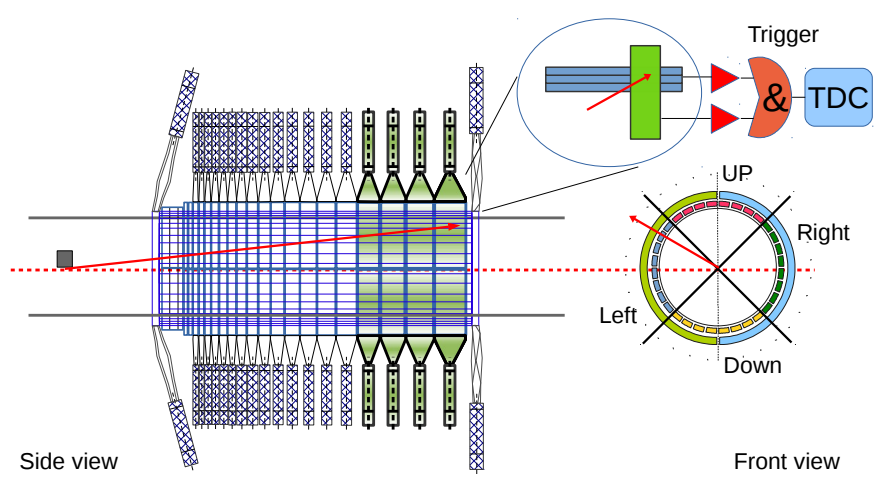

Figure 1: EDDA detector consists of the longitudinaly overlapping three angular plastic scintillator bars and transverse half rings. In EDM search only the colored part of the detector is used.

All the above-mentioned measurements were done using two none EDM dedicated detector systems. The EDDA detector depicted in the picture 1 and described in $[10,11]$. The original purpose of the detector was very different from EDM dedicated detector. But it was beneficial for its simplicity and was adopted for measurements. The trigger for the measurement has been newly developed, and it was the combination of both layers summed and grouped for up, down, left and right hits. The polar angular coverage was only 9 to 13 degree and full azimuthal coverage 
which nicely covers strong analyzing power range for deuteron carbon scattering around $250 \mathrm{MeV}$ kinetic energy [12]. Each trigger output (up, down, left and right) has been read with dedicated time stamping TDC, and in the same system, the accelerator RF cavity and RF solenoid signals were also monitored. All this analysed with dedicated online software tools give the experiment unprecedented flexibility to manipulate beam polarization and monitor it in the real time. The disadvantage of this system is mainly its restricted acceptance for the $d C$ reaction, the geometrical configuration of the plastic scintillating bars and due to old readout electronics, the reaction identification is not permanently monitored. Nevertheless, the results of the measurement were perfectly suited for the first phase of the experiments.

Due to the fact that former WASA [13] detector has been decommissioned from its original experimental program at COSY, we have modified it as a polarimeter. The only part of the complete detector which is interesting for the forward elastic scattering is shown in figure 2. Also, we have

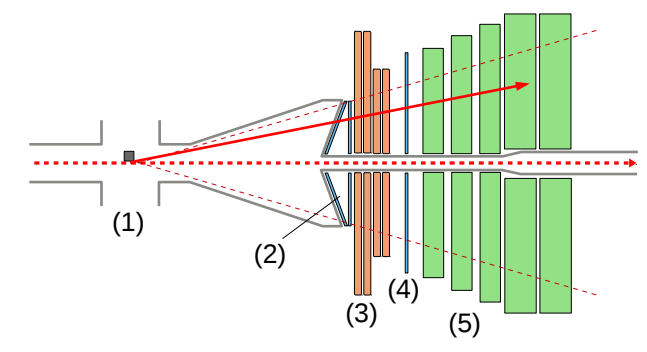

Side view

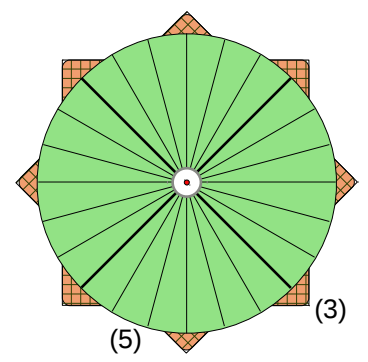

Front view

Figure 2: WASA as an polarimeter: (1)Target chamber; (2) Window counter; (3) Straw tubes; (4) Trigger hodoscope; (5) Range hodoscope;

modified its target section, and instead of frozen pellet target, we have modified target chamber with much bigger and universal vacuum flange. The same flange will be used for the EDM search dedicated polarimeter which is described later. In this chamber, we are using former ANKE strip targets, $25 \mu \mathrm{m}$ thick diamond and polyethylene targets. This was used to measure analyzing powers for deuteron carbon elastic scattering between $4^{\circ} \div 17^{\circ}$ of forward angle for seven different energies starting from $170 \div 370 \mathrm{MeV}$ and for pure vector, and tensor polarized beams. The polyethylene target will be used for the cross-section normalization for its hydrogen content. The vertical block target is also employed in a very similar way as we use at EDDA detector. The beam is vertically expanded using the white noise where the amplitude is proportionally moduled depending on count rate in the detector. This way the smooth beam extraction is guaranteed. The benefit of the former WASA system is its large $\Theta$ coverage $\sim 3 \div 17$ degrees and full $\phi$ coverage. The thick five layer range hodoscopes are capable of stopping deuterons and protons above $400 \mathrm{MeV}$ kinetic energy. This makes reaction identification very comfortable. It is also equipped with sampling ADC and very sophisticated straw tube tracking system.

Based on all acquired experience, we are developing the new concept $[14,15]$ for the dedicated polarimeter for storage ring EDM search. The general principle is to have very efficient, flexible and reliable system for the future experiments. For this purpose, we have postulated several requirements: 
- Precise reaction identification.

- Reliable functionality, short as well as long-term stability.

- Using modern electronics, we can eliminate dead time.

- Using novel scintillating material LYSO [20], we don't use strong magnetic fields for momentum measurement.

- The readout of scintillators is done using modern multi-pixel silicon photomultiplier arrays $[18,19]$, which eliminates high-voltage too.

- In front of crystals, we are testing plastic scintillating triangular bars for the $d E$ and $x, y$ sensitive tracking system. Also read out using SiPM's.

- We are developing innovative ballistic pellet target to have good vertex reconstruction and being quasi-non-invasive.

Up to now, only the carbon is considered as a scattering target material. It has good analyzing power for the elastic scattering and is ultra-high vacuum (UHV) compatible. One of the critical issue to explore is to compare different UHV compatible isotopes to optimize polarimeter efficiency. Figure

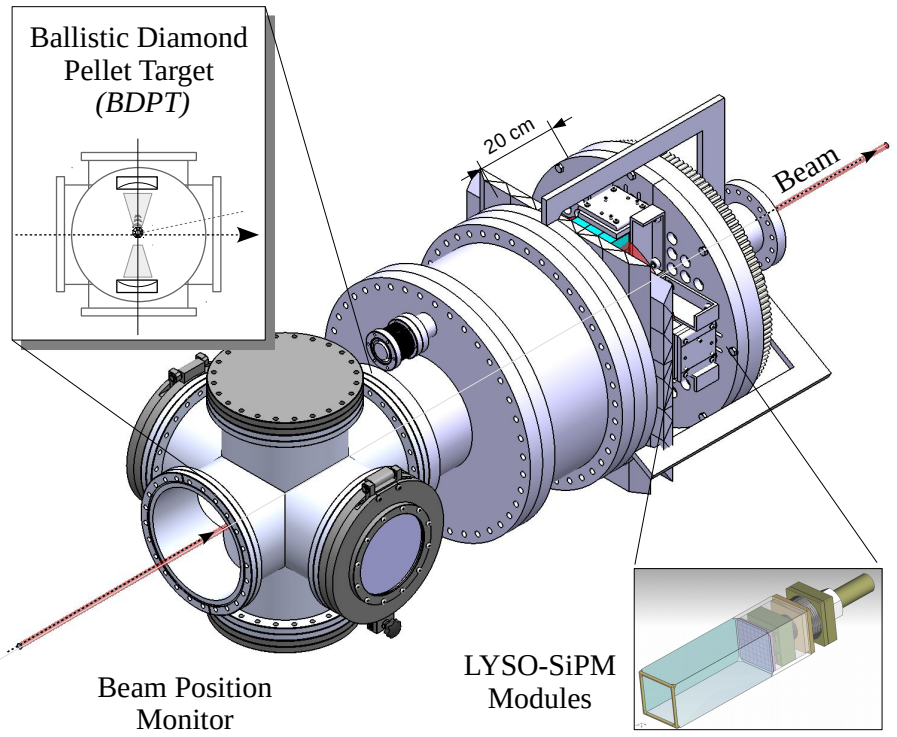

Figure 3: JEDI polarimeter. Left to right: Target flange where the beam position monitor Rogowsky coils and the ballistic pellet target will be mounter. In the middle part, the vacuum flight chamber with removable degrader will be installed. Next, the X, Y position sensitive plastic three angular bars ( $4 \mathrm{~cm}$ total thickness) are mounted. Finally, the detector part equipped with $8 \mathrm{~cm}$ long LYSO [20] crystals are assembled.

3 shows the technical drawing of the storage ring dedicated polarimeter. Figure 4 shows the existing 52 module setup at the beam position. The detector arms are sitting with $45^{\circ}$ degree inclination. In front of each arm, $2 \mathrm{~cm}$ thick plastic scintillator detector is mounted read with eight $6 \times 6$ silicon photomultipliers. 


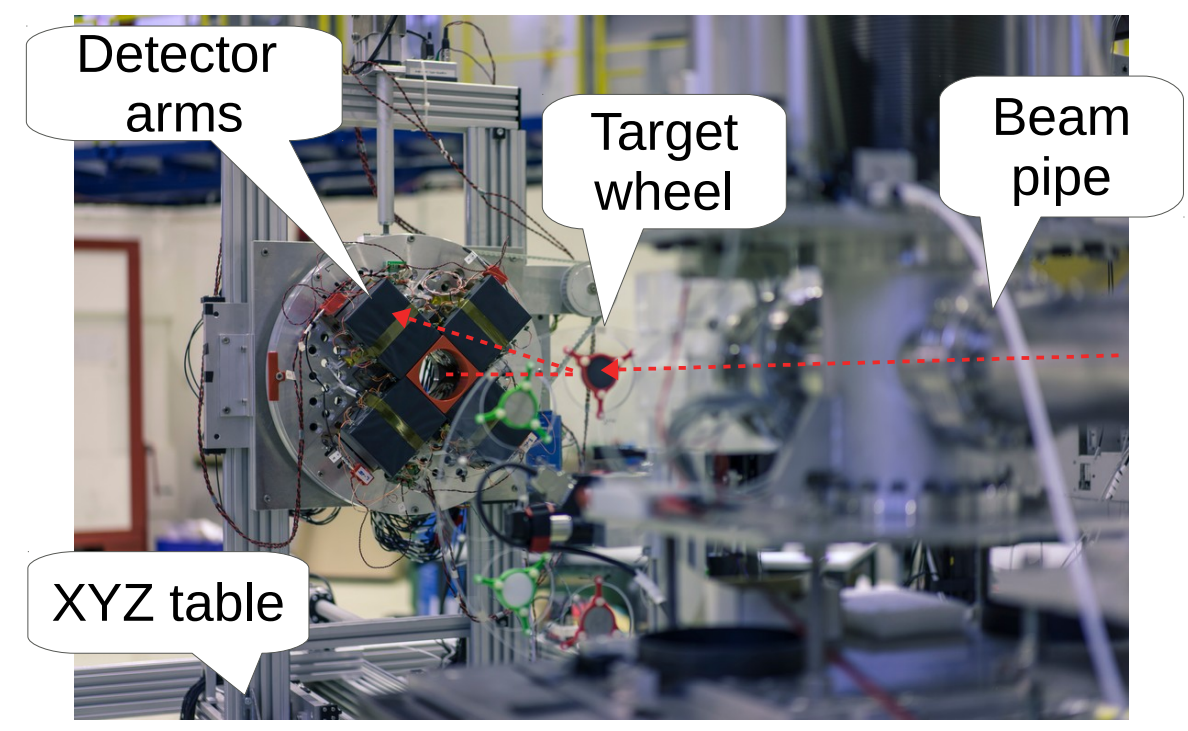

Figure 4: The detector part of JEDI polarimeter in the beam line at the external beam of COSY, inside the Big Karl experimental area.

Using this setup, we have tested all modules and did full energy and time calibration [16]. Using target weel, seven different target materials (Carbon, Polyethylene, Magnesium, Aluminum, Silicon, Nickel, and Tin) have been examined.

\section{DAQ and online analysis}

The significant part of the polarimeter is its sophisticated readout dead-time less system based on modern sampling ADC. The entire readout electronics is equipped with well established fast 250 MS/s 14 bit ADC from Struck [17] with a synchronised clock between modules and high-speed network readout $1 \mathrm{GBit} / \mathrm{s}$ per module (16 channels). For the last experiment, six modules have been used. In final setup, about ten modules, 160 channels will be directly read by the dedicated powerful server computer which is already in use. The multi CPU, each with multicore Intel Xeon server with Linux based system is employed to store and analyse online and offline data. Each signal has its time stamp and is divided into eight different integrals, done directly in the module onboard FPGA chip. This reduces the amount of data transfer from several thousand samples to just eight integrals in precisely chosen areas respective to an individual threshold for each channel. Two integrals are pre-trigger, used for pedestal subtraction as well as for the pre-signal detection. The case if previews signal is not fully gone and its tail is still affecting the current pedestal. Rest six integrals are fully integrating the charge generated by $8 \times 8$ SensL [18] SiPM array equipped with $3 \times 3 \mathrm{~mm}^{2}$ with $20 \mu \mathrm{m}$ pixels in total about $900 \mathrm{k}$ pixels per module. Figure 5 shows the system architecture how signals propagate from a detector to the online analysis computer. For the clean event selection, the two-dimensional histograms are filled, the energy loss into plastic scintillators versus deposited energy into the crystals. Figure 6 shows the typical picture how the elastic events are extracted. Different traces are also explained, there are three groups of events. Non-elastic deuterons are scattered elsewhere by losing a substantial amount of energy. Other groups of events are protons, and neutrons resulting break-up reaction on target and the horizontal line along the 


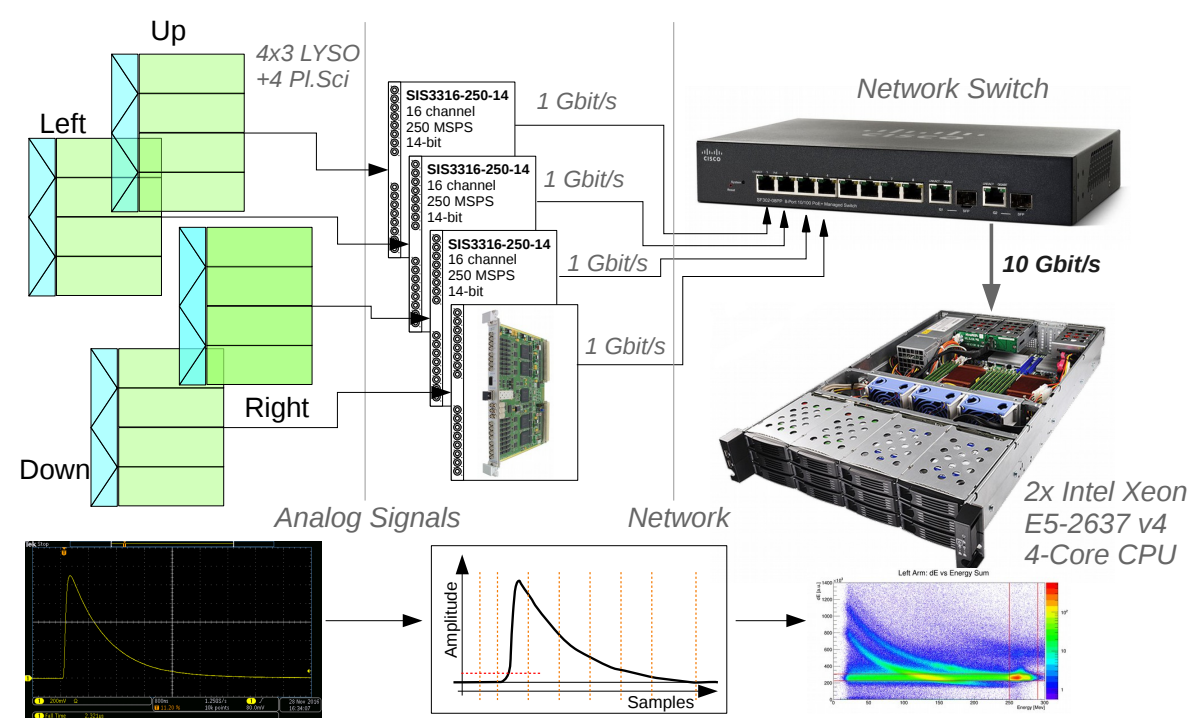

Figure 5: Data acquisition system for dedicated polarimeter. The connection between the experimental area and the detector is with $10 \mathrm{Gbit} / \mathrm{s}$ optical link.

elastic peak are events which were identified as the deuteron before the crystal, and it breaks inside the crystal. The efficiency of this events are measured as a function of the incoming deuteron energy and is reported [16].

\section{Summary}

As a summary, we would like to demonstrate the online measurement figure 7 which is a potent tool for the real-time polarization monitoring system. Here the results of left and right arms are shown for the solid $5 \mathrm{~mm}$ thick carbon (graphite) target. Left frame shows the stacked histogram of the real detector hits where the statistic for the inner crystals is significantly higher than for outside ones. That's why the excellent balance between statistics and analyzing power is needed. This is now as a so-called figure-of-merit FOM which is a product of cross-section by the squared
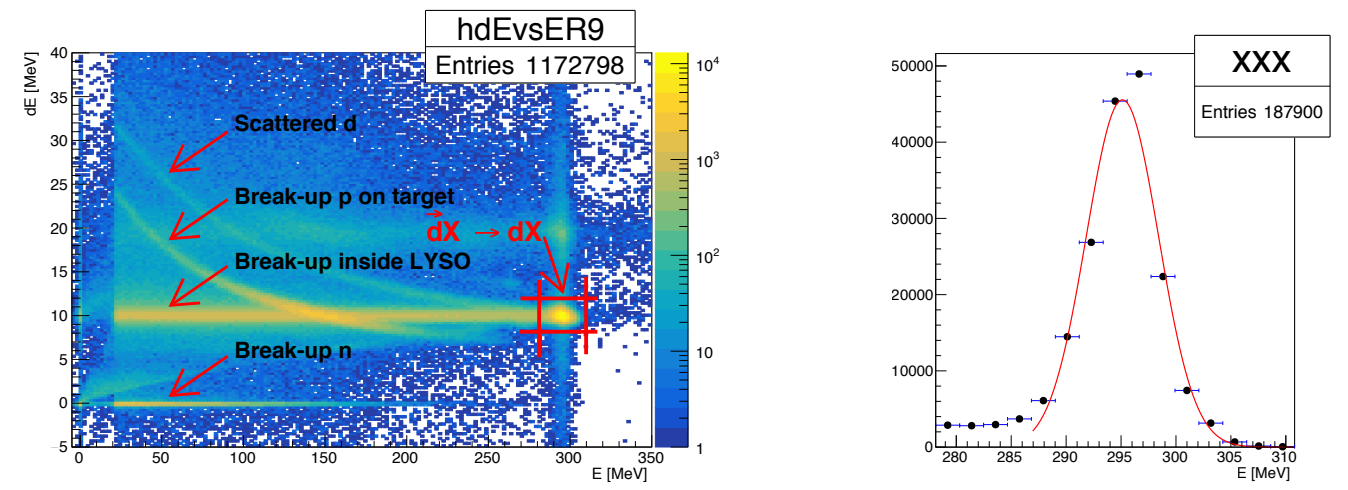

Figure 6: Left: $\Delta E$ vs. $E$ the red box shows the elastic peak of the deuteron. Right: extracted one dimensional histogram is shown for the event selection. 
analyzing power for the particular angle $F O M(\Theta)=\sigma_{e l}(\Theta) \times A_{y}^{2}$.

$$
C R(\theta)=\frac{\sqrt{N^{L \uparrow} N^{R \downarrow}}-\sqrt{N^{R \uparrow} N^{L \downarrow}}}{\sqrt{N^{L \uparrow} N^{R \downarrow}}+\sqrt{N^{R \uparrow} N^{L \downarrow}}} \approx P A_{y}(\Theta)
$$

The right frame (Fig.4.1) shows an online cross ration which is described in the equation 4.1 and is constructed to eliminate first-order systematic errors for the wrong beam and detector adjustments. As a matter of fact, in all our measurements we always use a sequence of one unpolarized and two vectors polarized (up, down) accelerator cycles.
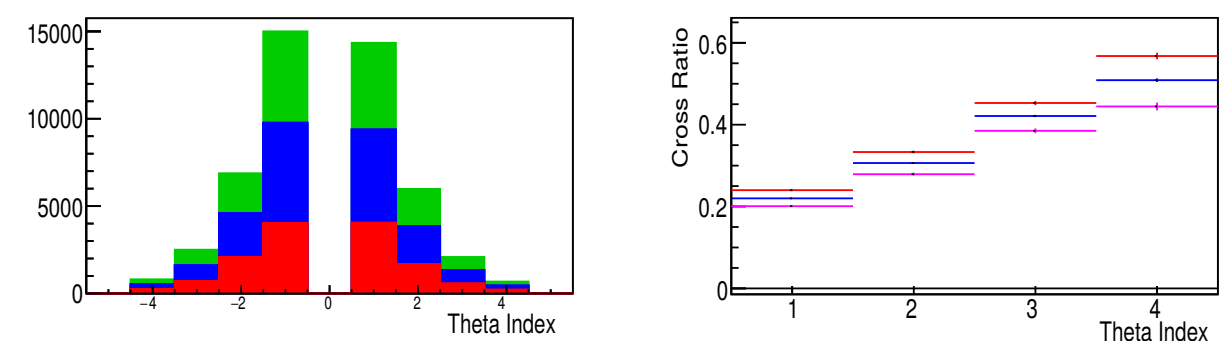

Figure 7: Online monitoring at $T_{d}=300 \mathrm{MeV}$ is shown. Left: The stacked histogram of left and right arms. Each with 12 crystals, $(4 \times 3)$ where indexing corresponds to $\Theta_{l a b}\left(4^{o} \div 10^{o}\right)$ angle. Right: The blue bars full cross ration, a combination of both polarized states. The red and pink bars the half cross rations, a comparison of each polarized states to unpolarized cycles.

As it was mentioned above, one of the objectives of the polarimeter development is to explore different target materials (isotopes). Figure 8 shows preliminary analyzing power for the incoming deuteron kinetic energy extracted from the online cross ration measurements by normalizing on average beam polarization of $40 \pm 10 \%$. The absolute polarization values might be quite different from the estimated one while the low energy polarimeter measurement has been done only at the beginning and end of the run. But, during the measurement time, runs are taken sequentially (the target is changed automatically after each run) the polarization value must be constant within $1 \%$ for each polarization state. The states are mostly entirely different, say $35 \%$ and $40 \%$. This figure (8) nicely demonstrates the difference between different materials for the same forward elastic scattering. The laboratory polar angle is very similar to what final polarimeter will get. In this experiments, we had a possibility to change the range by moving the platform upstream and downstream. Importantly the carbon shows a very significant advantage over other target materials. Whereas the silicon, tin, and aluminum could also be a very good choice. In this picture, the magnesium results are not shown while the runs were taken little bit later, but from our experience, it is very similar to aluminum and carbon as well. We have similar measurements for other energies as well and all analyzing powers, differential cross sections for different targets will be published soon.

\section{Acknowledges}

The authors wishes to acknowledge the support by the European Research Council via the ERC AdG srEDM (Contract number 694340). He also thanks all involved members of the JEDI collaboration and of the Institut für Kernphysik of Forschungszentrum Jülich for their dedication 


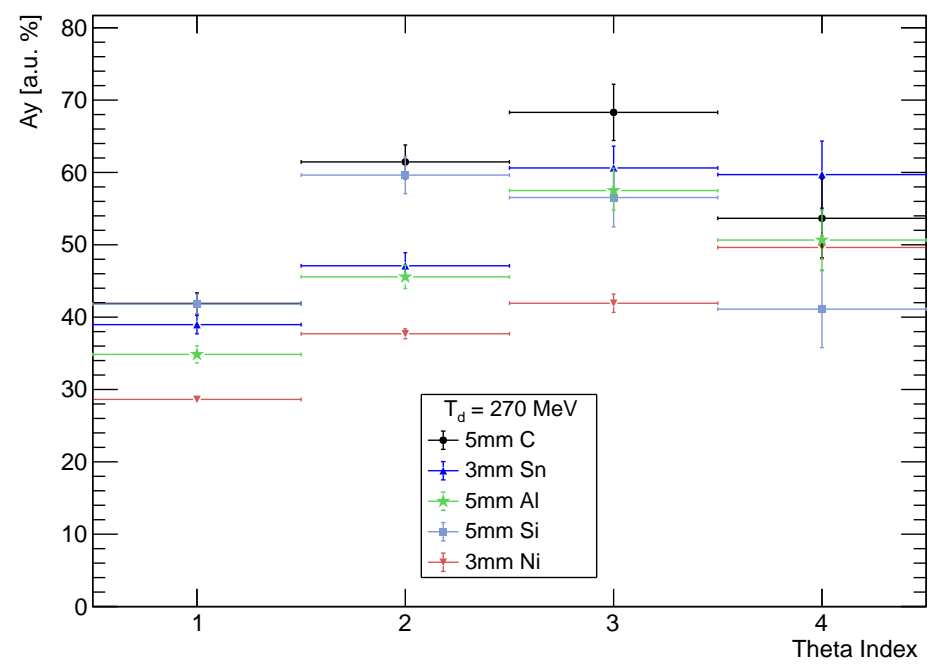

Figure 8: Preliminary online analysing powers at $T_{d}=270 \mathrm{MeV}$ are shown as a function of module index. Indexing of modules corresponds to $\Theta_{l a b}$ angles. NOTE: The absolute normalisation is very preliminary, but the comparison between target materials is more valuable. All these measurements are consequent runs within five hours of beam time. Here we can assume the beam polarisation and detector efficiency as constant.

and persistence towards this long-term project. This work has been financially supported by a grant from the Shota Rustaveli National Science Foundation of the Republic of Georgia (SRNSF Grant No. 217854, "A first-ever measurement of the EDM of the deuteron at COSY").

\section{References}

[1] JEDI collaboration web page http://collaborations.fz-juelich.de/ikp/jedi

[2] R. Maier et al., Nucl. Instrum. Methods Phys. Res., Sect. A 390, 1 (1997).

[3] D. Eversmann et al., Phys. Rev. Lett.115 (2015) 094801.

[4] G. Guidoboni et al., Phys. Rev. Lett.117 (2016) 054801.

[5] H. Hempelmann et al., Phys. Rev. Lett.119 (2017) 014801.

[6] A. Saleev, et al.,Phys. Rev. Accel. Beams 20, 072801 (2017).

[7] J. Slim et al., Nucl. Instrum. Methods Phys. Res., Sect. A(2016) 116.

[8] see http://sredm-ercgrant.de

[9] William M. Morse et al., Phys. Rev. ST Accel. Beams 16, 114001.

[10] D. Albers et al., Eur. Phys. J. A 22 , 125 (2004).

[11] J. Bisplinghoff et al., Nucl. Instrum. Methods Phys. Res., Sect. A329, 151(1993).

[12] Y. Satou et al., Phys. Lett. B 549, 307 (2002).

[13] Chr. Bargholtz et al., Nucl. Inst. Meth. A594 (2008). 
[14] Towards EDM Polarimetry, PoS(PSTP2015)026.

DOI: https://pos.sissa.it/243/026

[15] I. Keshelashvili et al., 2017 J. Phys.: Conf. Ser. 928012018 https://doi.org/10.1088/1742-6596/928/1/012018

[16] F. Müller et al., 2017 J. Phys.: Conf. Ser.928 012019 https://doi.org/10.1088/1742-6596/928/1/012019

[17] Struck SIS3316-250-14 16 channel 250 MSPS 14-bit http://www.struck.de/sis3316.html

[18] SensL ArrayJ-30020-64P-PCB http://sensl.com/products/sipmarrays/arrayj/

[19] KETEK PA3315-WB-0808 https://www.ketek.net/sipm/sipm-products/oem-arrays/

[20] Saint-Gobain, Cerium doped Lutetium PreLude420 crystal. http://www.crystals.saint-gobain.com/PreLude_420_Scintillatoraspx 